\title{
EL VALOR DEL SUELO HABITACIONAL
}

\section{THE VALUE OF THE HOUSING LAND}

\section{QUINTANA, Jesús}

Universidad de Sonora (UNISON)

Departamento de Ingeniería Civil y Minas

Profesor Investigador de Tiempo Completo

Blvd. Luis Encinas y Rosales S/N, Colonia Centro, Hermosillo, Sonora, C.P. 83000, México

Correo electrónico: jesus.quintana@unison.mx

Teléfono: +52 6622592183 y 84

\section{OJEDA, Arturo}

Universidad de Sonora (UNISON)

Departamento de Ingeniería Civil y Minas

Profesor Investigador de Tiempo Completo

Blvd. Luis Encinas y Rosales S/N, Colonia Centro, Hermosillo, Sonora, C.P. 83000, México

Correo electrónico: ojeda@dicym.uson.mx

Teléfono: +52 6622592183 y 84

\section{RAMÍREZ, Gerardo}

Universidad de Sonora (UNISON)

Departamento de Ingeniería Civil y Minas

Profesor Investigador de Tiempo Completo

Blvd. Luis Encinas y Rosales S/N, Colonia Centro, Hermosillo, Sonora, C.P. 83000, México

Correo electrónico: gerardo.ramirez@unison.mx

Teléfono: +52662 2592183 y 84

\section{SILVESTRE, José}

Universidad de Sonora (UNISON)

Departamento de Ingeniería Civil y Minas

Profesor Investigador de Tiempo Completo

Blvd. Luis Encinas y Rosales S/N, Colonia Centro, Hermosillo, Sonora, C.P. 83000, México

Correo electrónico: silvestre@dicym.uson.mx

Teléfono: +52 6622592183 y 84

Palabras Clave: Valor del suelo, consumo de agua, educación, renta, criminalidad

Key words: Land value, water consumption, education, rent, crime

\section{Resumen}

En una ciudad marcada por una evidente diferenciación del valor del suelo habitacional, este documento muestra las relaciones del valor y sus principales variables inmobiliarias, de acuerdo con el mercado de la ciudad de Hermosillo, Sonora, México. Lo anterior, a partir de conceptos tradicionales de deseabilidad del entorno edificado, de la accesibilidad urbana y de la jerarquía social; obtenida, en esta ocasión, a través de indicadores de educación, renta, accesibilidad, consumo de agua y criminalidad. El análisis se desarrolla a partir de una base de datos, geográficamente referenciada, con 427 mediciones de valores del suelo y sus características inmobiliarias, además de información del Instituto Nacional de Estadística y Geografía, mediciones del Organismo Operador Municipal Agua de Hermosillo y datos 
proporcionados por la Secretaría de Seguridad Pública del Estado de Sonora. Las medidas proporcionadas se integran en un sistema de información geográfica, con el cual es posible obtener modelos de precios hedónicos que permitan establecer relaciones estadísticas significativas. A partir, de análisis estadísticos y geoestadísticos multivariados, resueltos con la técnica de regresión lineal múltiple aproximada por OLS y la regresión geográficamente ponderada GWR, considerando una ponderación Gaussiana y una optimización de acuerdo al número de vecinos. Los resultados indican una importante asociación positiva del valor del suelo con el nivel de ingreso, el consumo de agua y la educación; y en este caso, la accesibilidad y la criminalidad en la ciudad parecen ser características que no aportan valor al suelo urbano habitacional.

\section{Abstract}

In a city marked by an evident differentiation of the value of the housing land, this document shows the relationships of the value and its main real estate variables, according to the market of the city of Hermosillo, Sonora, Mexico. The above, based on traditional concepts of desirability of the built environment, urban accessibility and social hierarchy; obtained, on this occasion, through indicators of education, rent, accessibility, water consumption and crime. The analysis is developed from a database, geographically referenced, with 427 measurements of land values and their real estate characteristics, in addition to information from the National Institute of Statistics and Geography, field measurements of the Municipal Agency Water Operator of Hermosillo and data provided by the Secretariat of Public Security of the State of Sonora. The measures provided are integrated into a geographic information system, with which it is possible to obtain hedonic price models that allow the establishment of significant statistical relationships. From statistical and geostatistical analyzes, multivariate, solved with the technique of multiple linear regression approximated by OLS and the geographically weighted regression GWR, considering a Gaussian weighting and an optimization according to the number of neighbors. The results indicate a significant positive association of land value with the level of income, water consumption and education; until this moment, the accessibility and the crime in the city, seem to be characteristics that do not contribute value to the residential urban land.

\section{Introducción}

La ciudad se conforma de suelo urbano, con distintos usos y distintas clasificaciones, es decir, dentro de la ciudad tenemos usos: habitacionales, comerciales, servicios e industriales; y cada uno de ellos, se clasifica en diferentes calidades, entendiendo por calidad, las características inmobiliarias del suelo que prefieren los consumidores, lo que lleva a tener suelos de alta, media y baja preferencia.

El objetivo de este documento es mostrar un procedimiento con el cual se estime el comportamiento del valor del suelo habitacional en la geografía de la ciudad de Hermosillo, Sonora, a partir del concepto de modelos de precios hedónicos que considere, además, posibles asociaciones espaciales de las variables inmobiliarias del mercado local.

Con lo cual se pretende verificar si, el valor del suelo urbano es resultado de una combinación de factores de educación, de ingreso familiar, de accesibilidad urbana, de criminalidad y de

Citación: QUINTANA, J. et al. El valor del suelo habitacional. En: Libro de proceedings, CTV 2018. XII Congreso Internacional Ciudad y Territorio Virtual. "Ciudades y Territorios Inteligentes". UNCuyo, Mendoza, 5-7 septiembre 2018. Barcelona: CPSV, 2018, p. 165-180. 
consumo de agua residencial; con lo cual sea posible explicar la formación actual del valor del suelo urbano habitacional.

La forma en que se produce y consume el recurso hídrico, está presente cada vez con mayor importancia en la economía, fue considerado por primera vez en la Cumbre de la Tierra en Río de Janeiro en 1992 y de Johannesburgo en el 2002; esto muestra la preocupación internacional por la sostenibilidad en los procesos de producción y consumo, de acuerdo con (Área de Gobierno de Medio Ambiente y Servicios de la Ciudad de Madrid, 2005).

El nivel de ingreso de las personas se relaciona directamente con las preferencias que están dispuestos a pagar, en el proceso de adquisición del suelo urbano de uso habitacional. Esto es, las personas con altos niveles de ingreso están en condiciones de pagar las mejores zonas para localizar su vivienda en la ciudad; donde se satisfagan la mayoría de sus preferencias, mientras que las personas con niveles de ingresos bajos, se deberán limitar en la cantidad de preferencias que les permitan sus ingresos acceder.

El proceso anterior genera una serie de asociaciones, donde las personas con niveles de ingresos altos, se ubican en las mejores zonas, mientras que las de menores ingresos van quedando agrupadas de acuerdo a su capacidad de compra.Estas asociaciones en primera instancia económica, genera nuevos deseos, como los de vivir cerca de personas con niveles de educación similares, problemas similares, mismas culturas, llegando incluso a la formación de agrupaciones con composiciones del mismo origen étnico.

La accesibilidad desde los primeros estudios de Von Thünen en Alemania, ha estado presente como un factor importante en el valor del suelo, a partir del modelo simplista de ciudad monocéntrica y homogénea, hasta sistemas más complejos con múltiples centros y características heterogéneas.

La criminalidad, es el resultado de hechos que transgreden las leyes y reglamentos, establecidos por el gobierno, son actitudes y acciones que lesionan a otras personas, sea esto en su integridad física, intelectual o en su patrimonio. La presencia de este tipo de acciones en una zona de la ciudad, es usualmente una característica que impacta negativamente en las preferencias de las personas. Si estas zonas no son preferidas por los demandantes de suelo urbano habitacional, provocan una baja en los precios de venta del suelo urbano, independientemente de otros tipos de atributos, como la accesibilidad, entorno urbano, etcétera.

El valor del suelo urbano, es resultado principal de una combinación de características como: la educación, el ingreso familiar, la accesibilidad urbana, la criminalidad y el consumo de agua; a partir de las cuales, es posible integrar modelos que expliquen su formación y comportamiento. El valor del suelo se estudia a partir de la formación espacial de los valores urbanos, que se define con base en la teoría económica espacial, donde es posible identificar tres componentes principales: la accesibilidad, las externalidades físicas y ambientales y los factores sociales.

\subsection{El consumo de agua}

En México, según datos del Consejo Nacional de Población (2010), se identificó que el $78 \%$ del total de habitantes es población urbana; y que, en Sonora, el $86 \%$ de la población ya está radicando en zonas urbanas en el estado; siendo que dicho proceso de asentamiento de la

Citación: QUINTANA, J. et al. El valor del suelo habitacional. En: Libro de proceedings, CTV 2018. XII Congreso Internacional Ciudad y Territorio Virtual. "Ciudades y Territorios Inteligentes". UNCuyo, Mendoza, 5-7 septiembre 2018. Barcelona: CPSV, 2018, p. 165-180. 
población en las localidades urbanas ha implicado fuertes presiones sobre el medio ambiente y las instituciones, lo cual es derivado del incremento de la demanda de los servicios urbanos.

En otro contexto, (Shandas \& Parandvash, 2009) desarrollan una investigación en Portland, Oregon, E.E.U.U. para cuantificar la influencia de zonas específicas y las características estructurales de desarrollos urbanos en el consumo de agua en una región metropolitana. Para ello, utilizaron Sistemas de Información Geográfica (SIG) para caracterizar 122,550 lotes de diferentes usos del suelo y estadísticamente asociadas a las formas urbanas y las características sociodemográficas de los usuarios del agua, con los datos empíricos sobre el consumo de agua durante un período de cinco años. Evaluaron el consumo de agua para las viviendas residencial unifamiliar (SFR), residencial multifamiliar (MFR), comercial, industrial y el uso del suelo a nivel de lote. En el análisis estadístico buscaron la asociación entre el consumo del agua y el uso del suelo para cada año, y posteriormente determinan el modelo utilizando regresión múltiple, efectuando diversas combinaciones con las variables independientes. Para el año 1999 encontraron una correlación fuerte y significativa entre el área de cada uso del suelo y el consumo de agua $\left(R^{2}=0.74\right)$. Para el año $2002-05$, las viviendas $S F R$, el uso comercial-industrial y el uso MFR son significativas, lo que ayuda a explicar el $55 \%$ a $64 \%$ del consumo total de agua.

\subsection{Criminalidad}

La criminalidad de acuerdo con (Cook, 1986), supone que los criminales y las víctimas son individuos racionales, que toman decisiones siguiendo procesos de optimización; esto es, que el criminal conoce las posibilidades de tener éxito o ser detenido, si tiene éxito las potenciales ganancias asociadas con el delito y en lo opuesto, los costos de ser detenido, el castigo o pena que le imponen las autoridades. Por otro lado, las víctimas también conocen las pérdidas potenciales que pueden tener en sus pertenencias y en este sentido incurren en gastos que aumenten las medidas de seguridad para prevenir el éxito de los delincuentes.

(Aguayo \& Chapa, 2012) en su trabajo de El robo a casa habitación en Monterrey, Nuevo León,encuentran que la localización de la vivienda y las características del vecindario son los elementos importantes para predecir la probabilidad del robo, como son las características de la vivienda y no así las características de sus ocupantes.

Por su parte (Urquijo, 2016), menciona que los datos de la Coordinación de Seguridad Ciudadana en Hermosillo, el $60 \%$ de los robos en casa habitación se llevan a cabo en los fraccionamientos tipo cerrada, sin diferenciar el nivel económico de los mismos. Esto fundamentalmente, por la poca atención en la prevención de estos delitos, pues el ciudadano confía en los vecinos de su cerrada.

De acuerdo con (Hindelang, Gottfredson, \& Garofalo, 1978) el robo de vehículos es un delito complejo de combatir, pues se manifiesta de diversas formas; es decir, se presenta el robo de motocicletas, vehículos particulares, transporte de pasajeros, de carga, etcétera. Además, los robos se pueden llevar a cabo, sin violencia o con violencia, con la víctima conduciendo, caminando hacia el vehículo o estacionándolo. Los robos se llevan a cabo en estacionamientos de centros comerciales, de diversión, de oficinas públicas, sobre las calles, en caminos vecinales o en carreteras.

En este sentido, (Vilalta, 2011) muestra que el robo de vehículo es un fenómeno que se presenta con una lógica geográfica y temporal, por lo que es un delito que puede ser

Citación: QUINTANA, J. et al. El valor del suelo habitacional. En: Libro de proceedings, CTV 2018. XII Congreso Internacional Ciudad y Territorio Virtual. "Ciudades y Territorios Inteligentes". UNCuyo, Mendoza, 5-7 septiembre 2018. Barcelona: CPSV, 2018, p. 165-180. 
combatido. Esto es, que es posible identificar los lugares más frecuentados por estos delincuentes y los momentos en que prefieren actuar.

El narcomenudeo es un fenómeno normalmente asociado al narcotráfico, como parte de su estructura final para llegar al consumidor. Es la parte perceptible por los ciudadanos en la compraventa y consumo de sustancias ilegales relacionadas con la alteración de la conducta de los individuos y normalmente suele tener una estructura territorial dominada por grupos delincuenciales organizados.

Por su parte (Cortés \& Parra, 2011) caracteriza el mercado del narcomenudeo a partir de tres aspectos básicos: puntos de venta, la monetización y el consumo. Esto es, el narcomenudeo es una actividad ilegal, que con el tiempo genera espacios y actitudes al desarrollo de otro tipo de actividades ilegales, como el robo, la extorsión, la deserción escolar y la violencia intrafamiliar y urbana.

\section{Metodología}

\subsection{Descripción del caso de estudio}

El Estado de Sonora se localiza al noroeste de México, en la zona más árida del territorio nacional conocida como Desierto de Sonora que se extiende al sur de Estados Unidos de América a través de los estados de Arizona y California, y en México por el Estado de Baja California y Sonora. La ciudad de Hermosillo, capital del estado se localizada a $29^{\circ} 05^{\prime} 56^{\prime \prime} \mathrm{N}$ y $110^{\circ} 57^{\prime} 15$ ”O en el noroeste de la República Mexicana y a $289 \mathrm{~km}$ de la frontera con los Estados Unidos de América (¡Error! No se encuentra el origen de la referencia.).

La ciudad con una altitud promedio de $216 \mathrm{MSNMM}^{1}$, una topografía sensiblemente plana, una mancha urbana de $160.93 \mathrm{~km}^{2}$, donde $125.68 \mathrm{~km}^{2}$ corresponden a superficies de lotes y predios urbanos, de los cuales el $37.25 \%$ se encuentran baldíos (Instituto Municipal de Planeacion Urbana, 2006). De donde es posible inferir la flexibilidad de la autoridad municipal en el planeamiento del desarrollo de la ciudad, lo cual es aprovechado por algunos sectores de la población para mantener de cierta forma el poder adquisitivo de su dinero y obtener una renta importante (plusvalía) a costa de las inversiones públicas en servicios y los costos que el resto de la población tiene que pagar al recorrer cada vez mayores distancias del hogar al trabajo, escuela o comercio.

La ciudad de Hermosillo registra una población de 715,061 habitantes y un stock de vivienda compuesto por 213,304 viviendas particulares habitadas, donde el $95.24 \%$ posee agua entubada, el $94.59 \%$ sistema de drenaje y el $97.49 \%$ servicio de energía eléctrica (Instituto Nacional de Estadística Geografía, 2010).

La ciudad presenta zonas de valores de suelo habitacional muy diferentes, como es la región central de la ciudad, compuesta por el antiguo casco de la ciudad, donde se localiza principalmente comercio de segundo nivel. De este centro de atracción se desprenden varías vías de comunicación, como los bulevares Francisco Eusebio Kino, Morelos, Luis Encinas, Colosio, Navarrete, entre otros, mismos que se muestran en la ¡Error! No se encuentra el origen de la referencia.. En estas vialidades se generan zonas comerciales de primer,

1 MSNMM metros sobre el nivel medio del mar.

Citación: QUINTANA, J. et al. El valor del suelo habitacional. En: Libro de proceedings, CTV 2018. XII Congreso Internacional Ciudad y Territorio Virtual. "Ciudades y Territorios Inteligentes". UNCuyo, Mendoza, 5-7 septiembre 2018. Barcelona: CPSV, 2018, p. 165-180. 
segundo y tercer nivel, que marcan el crecimiento urbano de la ciudad. Las áreas comprendidas entre estas vialidades forman entornos habitacionales de alto, medio y bajo nivel; donde parece existir una asociación espacial de niveles altos con altos y bajos con bajos, con transiciones graduales.

Figura 1. Localización de Hermosillo

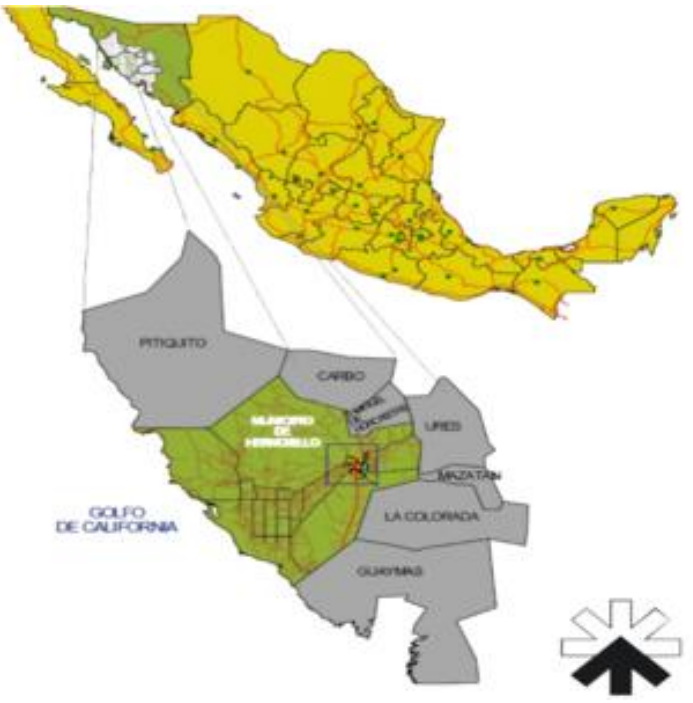

Fuente: Fuente: Inst. Mun. de Planeación Urbana (2016)

\section{Figura 2. Mancha Urbana de Hermosillo}

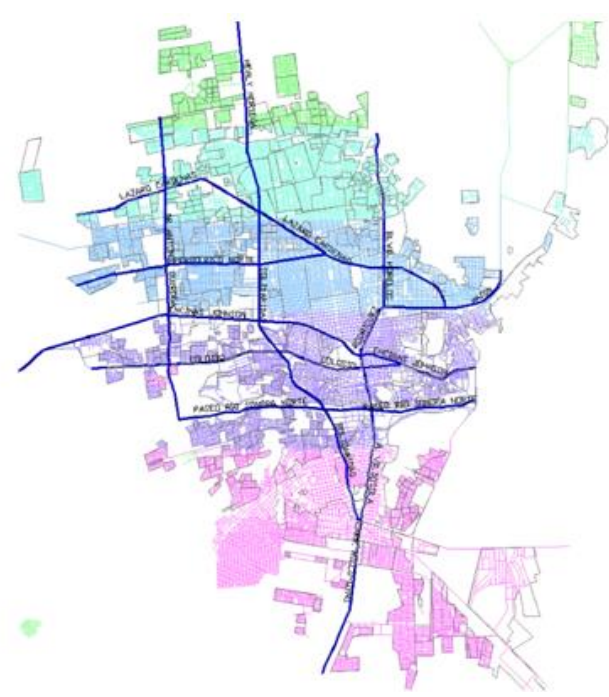

Fuente: Elaboración propia

Hermosillo, una urbanización de tamaño medio en el país, con un enfoque hacia los servicios, con una industria poco desarrollada, con pocas posibilidades de actividades primarias, debido a su limitante hídrica. Presenta un rango de valores de suelo que van de los $\$ 65.00 / \mathrm{m} 2$, hasta valores de $\$ 4,500.00 / \mathrm{m} 2$ (Colegio de Valuadores Profesionales del Estado de Sonora, 2013).

\subsection{Diseño de la investigación}

Se recolectó directamente en campo mediante recorridos por la ciudad, localizando los espacios de suelo habitacional disponibles, de ahí se conformó en gabinete una muestra de valores del suelo urbano habitacional unifamiliar, utilizando una hoja electrónica en Excel. Además de los datos de campo, se colectó información de diferentes bases de datos de sitios en internet publicadas por empresas dedicadas a la compraventa de bienes raíces, asesores de crédito y personas físicas.

De igual forma se llevó a cabo la recolección de información consultando las bases de datos utilizados en sistemas de gestión de captura de avalúos en unidades de valuación acreditadas en la Sociedad Hipotecaria Federal, en las cuales se almacena la información utilizada en la realización de los diferentes trabajos de valuación, como comparables de ofertas de mercado. Finalmente se logra integrar 427 casos de valores unitarios de terrenos urbanos habitacionales.

Por otro lado, la variable FAC_AGUA consumo de agua doméstica, se integra a partir de información del Organismo Operador Agua de Hermosillo, con información de los consumo de agua potable generados en las viviendas para el año 2015, con lo que se obtiene el consumo medio mensual, representativo de los doce meses del año para cada una de las viviendas pertenecientes a las colonias de la ciudad, de acuerdo con el procedimiento establecido en (Ojeda A. , 2013).

Citación: QUINTANA, J. et al. El valor del suelo habitacional. En: Libro de proceedings, CTV 2018. XII Congreso Internacional Ciudad y Territorio Virtual. "Ciudades y Territorios Inteligentes". UNCuyo, Mendoza, 5-7 septiembre 2018. Barcelona: CPSV, 2018, p. 165-180. 
En la ¡Error! No se encuentra el origen de la referencia. se muestran los distintos valores de consumo de agua en la geografía de la ciudad, observándose zonas como Los Lagos y áreas cercanas al Bulevar Morelos que presentan altos consumos de agua y se asocian con valores de suelos altos; y los menores consumos, se presentan en zonas periféricas al norte, sur y noroeste, características de valores de suelos bajos.

\section{Figura 3. Distribución de los consumos de agua}

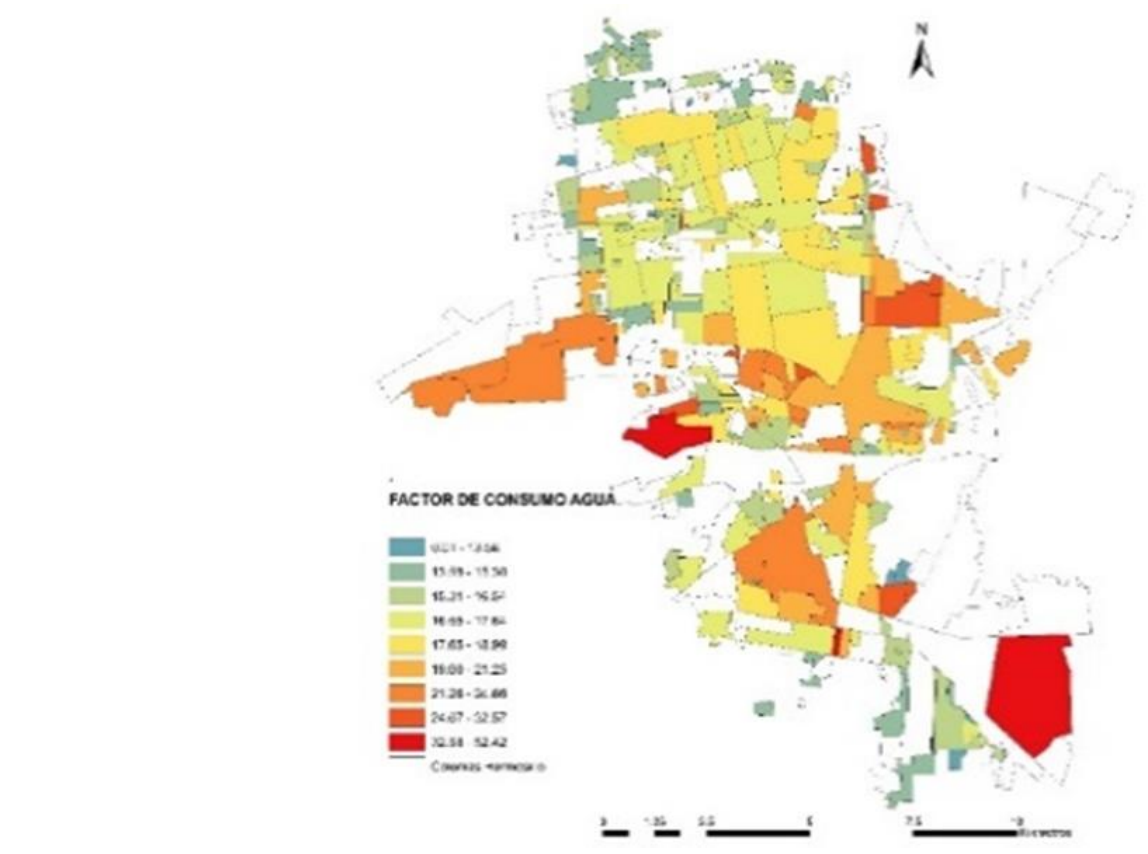

Fuente: Elaboración propia

FAC_CRIM criminalidad, es una variable que se integra a partir de información que proporciona la Secretaría de Seguridad Pública del Gobierno del Estado de Sonora, en su portalhttp://sspsonora.gob.mx/, donde se consideran los delitos registrados (Secretaría de Seguridad Pública, 2016): narcomenudeo, robo de vehículo, robo a casa habitación, robo a comercio, lesiones dolosas.

Esta información se utiliza para obtener un factor que integre esta información, para lo cual se aplica el análisis estadístico de reducción de dimensiones, en particular el Análisis Factorial aplicando el método de Componentes Principales, usando el SPSS 22. Los resultados muestran una extracción muy cercana a la unidad y una varianza total explicada de $63.2 \%$.

El comportamiento del factor de criminalidad a partir de estas cuatro variables, se muestra en la ¡Error! No se encuentra el origen de la referencia.. Donde es posible observar, zonas como el Centro de la ciudad, junto con colonias como el Sahuaro, Solidaridad y el Palo Verde con alta incidencia de este tipo de delitos; y en menor grado colonias como la Balderrama, San Benito, Altares, Ley 57, Las Lomas, Nuevo Hermosillo, entre otras; dejando a gran parte de la ciudad con indicadores bajos en el aspecto delictivo. FAC_ACC accesibilidad, es un componente obtenido de información de campo y cálculos de (Morales, 2015), para integrar una medida de accesibilidad en la ciudad de Hermosillo, a partir de las tres variables siguientes: accesibilidad en distancia, accesibilidad en tiempo, accesibilidad en costo.

Citación: QUINTANA, J. et al. El valor del suelo habitacional. En: Libro de proceedings, CTV 2018. XII Congreso Internacional Ciudad y Territorio Virtual. "Ciudades y Territorios Inteligentes". UNCuyo, Mendoza, 5-7 septiembre 2018. Barcelona: CPSV, 2018, p. 165-180. 
Se reducen las dimensiones, utilizando el Análisis Factorial, donde se obtiene una extracción del 95 al 99\%, con una varianza total explicada del 97.7\%. Finalmente, el comportamiento del factor de accesibilidad obtenido a partir de estas tres medidas, se muestra en la ;Error! No se encuentra el origen de la referencia.. Donde es posible observar, como de forma natural, las partes cercanas al centro de la ciudad comparten factores de accesibilidad importantes y de forma gradual van disminuyendo conforme se aleja del centro de la ciudad.

Figura 4. Distribución de la criminalidad

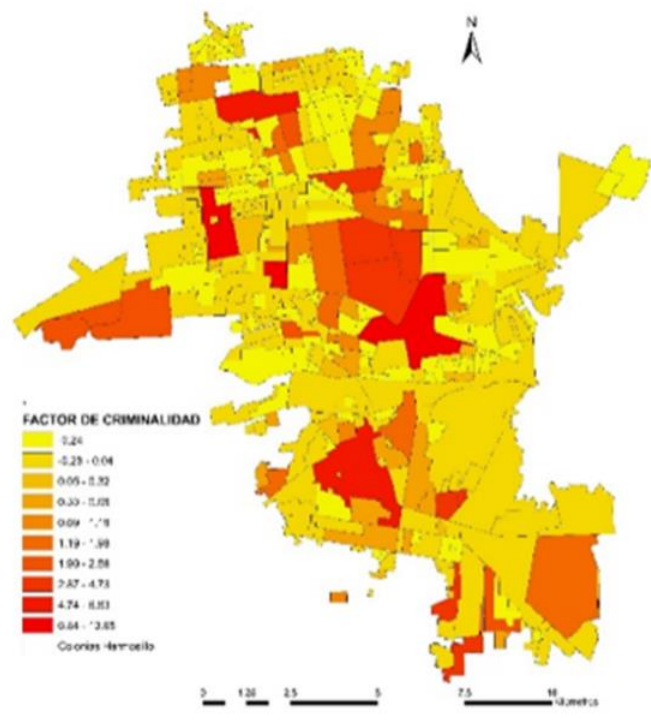

Fuente: Elaboración propia

\section{Figura 5. Distribución de la accesibilidad}

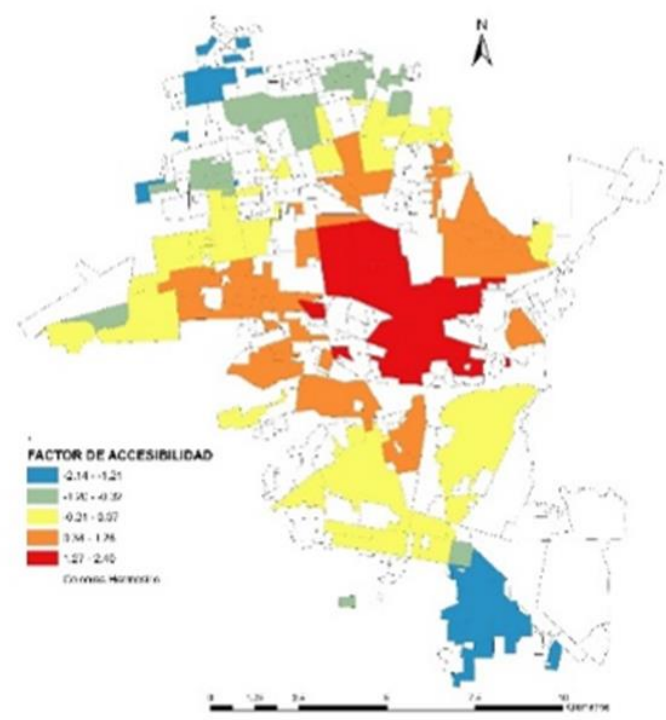

Fuente: Elaboración propia

FAC_REN la renta, es la estimación del indicador de ingresos y se obtiene a partir de variables económicas y de viviendas, derivadas de (Instituto Nacional de Estadística Geografía, 2010), como la población ocupada (ECO4), población ocupada de 12 años y más con al menos un grado aprobado de educación superior o posgrado (ECO22), promedio de ocupantes por cuarto (VIV5) y viviendas particulares habitadas que disponen de internet (VIV36).

Se reducen las dimensiones, utilizando el Análisis Factorial. En este proceso se excluye la variable ECO4que presenta una pobre integración y poca varianza explicada; una vez realizado esto, el componente final presenta extracciones del 80 al $84 \%$ y una varianza total explicada de 82.3\%. Finalmente, el comportamiento del factor de renta obtenido a partir de estas tres variables, se muestra en la ¡Error! No se encuentra el origen de la referencia.. Donde es posible observar valores de renta alta en la zona Centro, Los Lagos y sus alrededores, así como la zona del Bulevar Morelos, con acentuación hacia el Cerro del Bachoco; mientras que los valores de renta baja se presentan fundamentalmente en la periferia, con excepción de las terminaciones de los Bulevares Colosio y Morelos.

FAC_EDU educación, es la estimación del indicador de educación, a partir de las variables de (Instituto Nacional de Estadística Geografía, 2010), que se listan en seguida: población de 15 años o más con educación pos-básica (EDU40), población de 25 años y más con al menos un grado aprobado de educación superior (EDU46), grado promedio de escolaridad (EDU49).

Se reducen las dimensiones, utilizando el Análisis Factorial. Los resultados muestran una extracción de 95 al $98 \%$ y una varianza total explicada de $96.6 \%$. 
Finalmente, el comportamiento del factor de educación obtenido a partir de estas tres variables, se muestra en la ¡Error! No se encuentra el origen de la referencia., donde, como el anterior, presenta zonas de educación altas en Los Lagos y sus alrededores, llegando hasta el centro de la ciudad, así como en el Bulevar Morelos y alrededores hacia el cerro del Bachoco; sin embargo los valores de educación más bajos se encuentran situados hacia las periferias propias de viviendas autoconstruidas, con asentamientos de hace unos 20 años o más.

Figura 6. Distribución de la renta

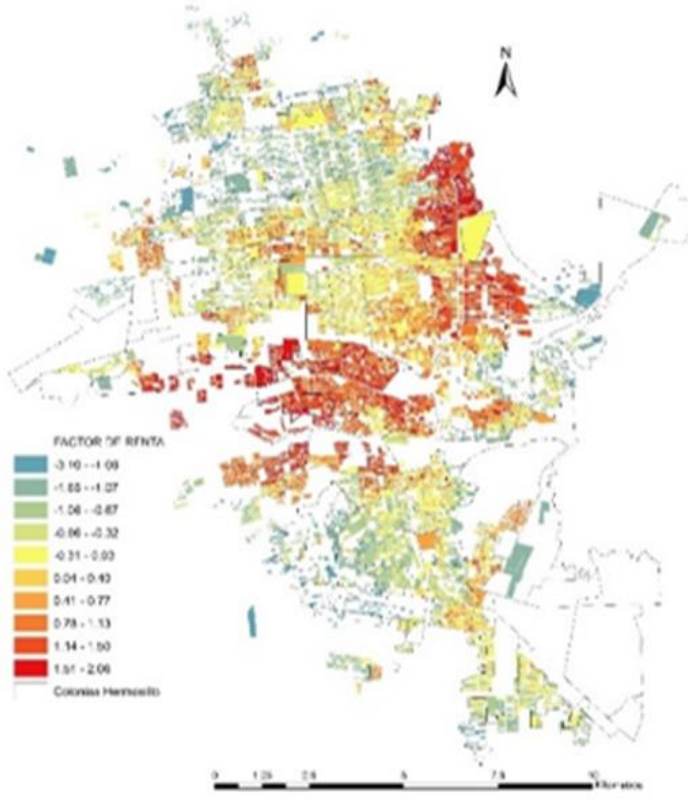

Fuente: Elaboración propia
Figura 7. Factor de la educación

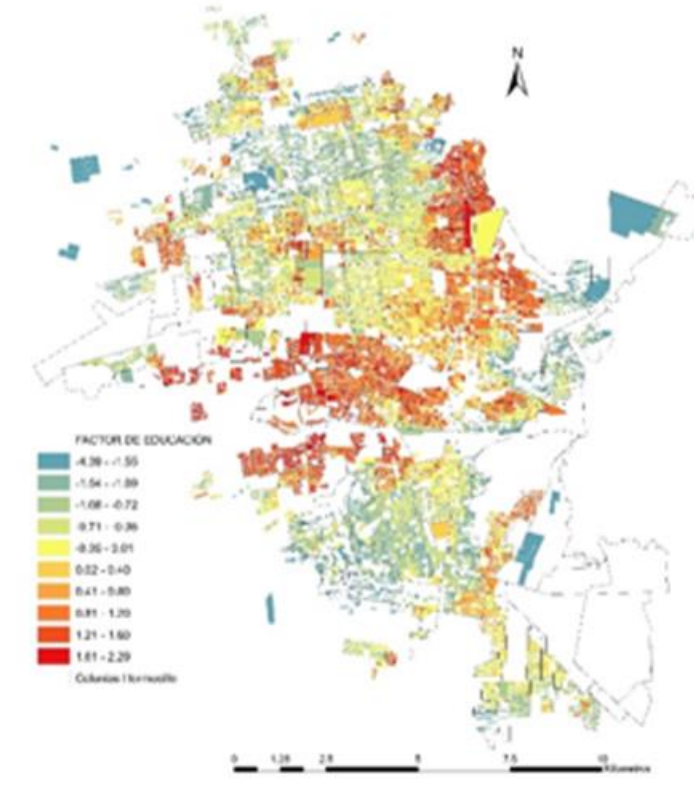

Fuente: Elaboración propia

Una vez integrados los cinco factores descritos, se lleva a cabo un análisis estadístico multivariado ordinario y geoespacial (OLS y GWR, en inglés) mediante el uso de SPSS 22 y ArcGis 10.2, con los cuales se procede a explicar las relaciones existentes entre los valores del suelo y los factores de mayor influencia. Previo a esto, se revisa la normalidad de la variable dependiente V_U_SUELO, determinando la situación de datos atípicos, posibles (outliers). Seguidamente se revisa el comportamiento normal de la variable dependiente aplicando la prueba de Kolmogorov-Smirnov (K-S) para verificar la "bondad de ajuste" que permite medir el grado de concordancia existente entre la distribución de un conjunto de datos y una distribución de probabilidad de tipo normal.

Ahora bien, la exhaustiva recolección de datos del valor del suelo habitacional y la diversidad de sus datos produce una concentración de información sesgada, por lo que es necesario realizar una reducción de los casos en exceso. Esto exige una reducción aleatoria en los rangos de alta concentración de casos, hasta obtener un comportamiento normal en la variable dependiente y su transformación de los datos con el LN_V_U_SUELO. Por lo que, de acuerdo a esto, en el análisis exploratorio de la prueba K-S se obtiene un p-valor $=0.048$, lo cual es estadísticamente significativo puesto que para ser acepado, el p-valor obtenido deberá ser mayor o igual a 0.05 , lo cual se considera suficiente y se puede afirmar que no existen problemas importantes de normalidad en la variable dependiente transformada, Tabla 1.

Citación: QUINTANA, J. et al. El valor del suelo habitacional. En: Libro de proceedings, CTV 2018. XII Congreso Internacional Ciudad y Territorio Virtual. "Ciudades y Territorios Inteligentes". UNCuyo, Mendoza, 5-7 septiembre 2018. Barcelona: CPSV, 2018, p. 165-180. 
Tabla 1. Prueba de Kolmogorov-Smirnov para una muestra

\begin{tabular}{llrrr}
\hline & & V_U_SUELO & LOG_V_U_SUELO & LN_V_U_SUELO \\
\hline $\mathrm{N}$ & & 218 & 218 & 218 \\
Parámetros normales ${ }^{\text {a,b }}$ & Media & 1493,694771 & 3,0921 & 7,1198 \\
& Desv. típica & 940,0833229 &, 62506 &, 092 \\
Diferencias más extremas & Absoluta &, 137 &, 092 &, 092 \\
& Positiva &, 137 &, 092 &,- 057 \\
& Negativa &,- 113 &,- 057 & 1,366 \\
Z de Kolmogorov-Smirnov & & 2,016 & 1,366 &, 048 \\
Sig. asintót. (bilateral) &, 001 &, 048 & \\
\hline
\end{tabular}

a. La distribución de contraste es la Normal.

b. Se han calculado a partir de los datos.

Fuente: Elaboración propia

Lo anterior reduce la cantidad de casos de 427 datos del mercado inmobiliario del suelo, a una muestra normalmente distribuida de 218 casos, donde ya es posible llevar a cabo procedimientos como la regresión lineal múltiple, en la solución de modelos de precios hedónicos, que expliquen el comportamiento del valor del suelo a partir de los factores presentados.

En la ¡Error! No se encuentra el origen de la referencia., se muestra el histograma de distribución de frecuencias observando su parecido el cumplimiento de la normalidad de los datos de la variable dependiente transformada LN_V_U_SUELO.

Figura 8. Histograma de frecuencias del LN_V_U_SUELO

Fuente: Elaboración propia

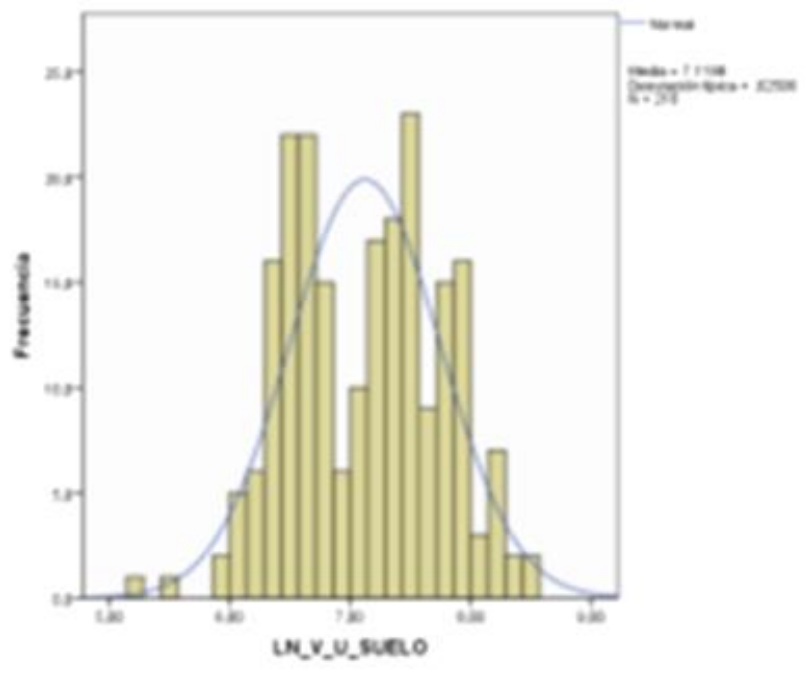

\section{Resultados}

\subsection{Análisis estadístico simple}

El primer análisis se realiza explicando el valor del suelo (LN_V_U_SUELO), a partir de cada uno de los factores por separado, obteniéndose los resultados de la Tabla 2, donde se observa explicaciones de $\mathrm{R}^{2}$ muy importantes en los factores de educación, consumo de agua y renta.

Citación: QUINTANA, J. et al. El valor del suelo habitacional. En: Libro de proceedings, CTV 2018. XII Congreso Internacional Ciudad y Territorio Virtual. "Ciudades y Territorios Inteligentes". UNCuyo, Mendoza, 5-7 septiembre 2018. Barcelona: CPSV, 2018, p. 165-180. 
Tabla 2. Explicación del valor del suelo

\begin{tabular}{ll}
\hline FACTOR & $\mathrm{R}^{2}$ \\
\hline De educación & $49.0 \%$ \\
De renta & $58.8 \%$ \\
De accesibilidad & $23.6 \%$ \\
De consumo de agua & $49.0 \%$ \\
De criminalidad & $12.4 \%$ \\
\hline
\end{tabular}

Fuente: Elaboración propia

En la Figura 1 se muestran los gráficos de dispersión del LN_V_U_SUELO y los factores, donde por si solos, cada uno de estos factores presentan explicaciones del logaritmo natural del valor del suelo, con $\mathrm{R}^{2}$ que oscilan del $12.4 \%$ al $58.8 \%$.

Figura 1. Gráficos de dispersión de los factores y el In del valor del suelo
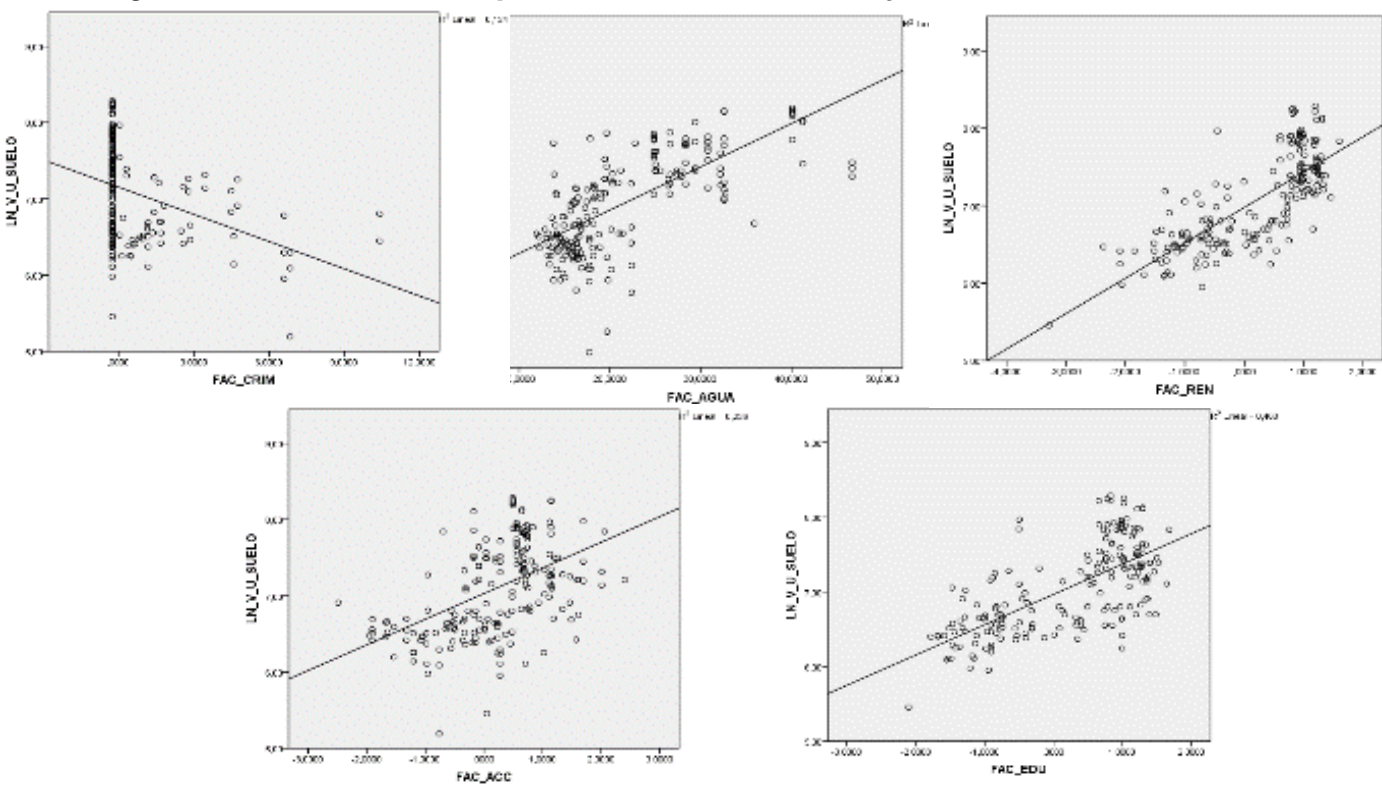

Fuente: Elaboración propia

\subsection{Modelo de Precios Hedónicos resuelto con estadística multivariada OLS}

Se plantea el modelo de precios hedónico y se resuelve con la técnica de regresión lineal múltiple, en el software SPSS22, introduciendo el LN_V_U_SUELO como la variable dependiente y los cinco factores como las variables independientes, se procede a la solución del sistema aplicando como criterio de optimización, los mínimos cuadrados ordinarios (OLS), en un proceso de pasos sucesivos, con una probabilidad $F$ de Snedecor de entrada 0.5 y de salida de 0.10 . Los resultados se muestran en la Tabla 3.

Tabla 3. Coeficientes ${ }^{\text {a }}$

\begin{tabular}{|c|c|c|c|c|c|c|c|c|c|c|c|}
\hline \multirow{2}{*}{\multicolumn{2}{|c|}{ Modelo }} & \multicolumn{2}{|c|}{$\begin{array}{l}\text { Coeficientes no } \\
\text { estandarizados }\end{array}$} & \multirow{2}{*}{$\begin{array}{c}\begin{array}{c}\text { Coeficientes } \\
\text { tipificados }\end{array} \\
\text { Beta }\end{array}$} & \multirow[b]{2}{*}{$t$} & \multirow[b]{2}{*}{ Sig. } & \multicolumn{3}{|c|}{ Correlaciones } & \multicolumn{2}{|c|}{$\begin{array}{l}\text { Estadísticos de } \\
\text { colinealidad }\end{array}$} \\
\hline & & B & Error típ. & & & & $\begin{array}{l}\text { Orden } \\
\text { cero }\end{array}$ & Parcial & $\begin{array}{l}\text { Semi } \\
\text { parcial }\end{array}$ & Tolerancia & FIV \\
\hline \multirow[t]{3}{*}{2} & (Constante) & 6,333 & 081 & & 78,342 & 000 & & & & & \\
\hline & FAC REN & ,319 & ,029 &, 535 & 11,021 & ,000 & ,767 & ,623 & ,438 & ,672 & 1,488 \\
\hline & FAC_AGUA & ,031 & ,004 & ,405 & 8,352 &, 000 & ,711 &, 517 & ,332 & ,672 & 1,488 \\
\hline
\end{tabular}

a. Variable dependiente: LN_V_U_SUELO

Fuente: Elaboración propia

Citación: QUINTANA, J. et al. El valor del suelo habitacional. En: Libro de proceedings, CTV 2018. XII Congreso Internacional Ciudad y Territorio Virtual. "Ciudades y Territorios Inteligentes". UNCuyo, Mendoza, 5-7 septiembre 2018. Barcelona: CPSV, 2018, p. 165-180. 
Las variables reconocidas en el proceso corresponden a FAC_REN y FAC_AGUA, obteniendo una explicación de $\mathrm{R}^{2}$ corregida de $69.5 \%$, un error de 0.333 , un Durbin Watson de 1.857 , muy cercano al 2 que representa la nula existencia de correlación de los residuos del modelo, el análisis de la varianza con significancia muy cercana a cero, representativa de una fuerte correlación lineal de la variable dependiente con las independientes del modelo.

De igual forma se revisa que los residuos presenten media cero y una desviación estándar cercana a la unidad y que el modelo no presente indicios de hetereocedasticidad. Lo anterior muestra que el valor del suelo se asocia fuertemente, en primera instancia, con zonas donde las personas presentan altos ingresos; y en segundo lugar, con consumidores de volúmenes altos de agua. La ecuación muestra de forma más explícita estas relaciones.

$$
L N V U_{\text {SUELO }}=6.333+0.319 F A C_{\text {RENTA }}+0.031 * F A C_{\text {AGUA }}
$$

\subsection{Modelos de Precios Hedónicos con geoestadística multivariada GWR}

Una vez obtenido el modelo estadístico que define el valor del suelo, a partir de los factores inmobiliarios, es necesario revisar posibles asociaciones espaciales de los indicadores del valor del suelo y del valor mismo. Para esto se obtiene un mapa temático de los residuos del modelo estadístico obtenido, mismo que para efectos de visualización se interpola en la mancha urbana de la ciudad, resultado que se muestra en la ¡Error! No se encuentra el origen de la referencia..

Donde se observa que los residuos altos se asocian con otros residuos altos y los bajos con bajos, síntoma de una posible asociación espacial de los residuos del modelo, por lo que es conveniente revisar formalmente este fenómeno.

Figura 10. Distribución espacial de los residuos

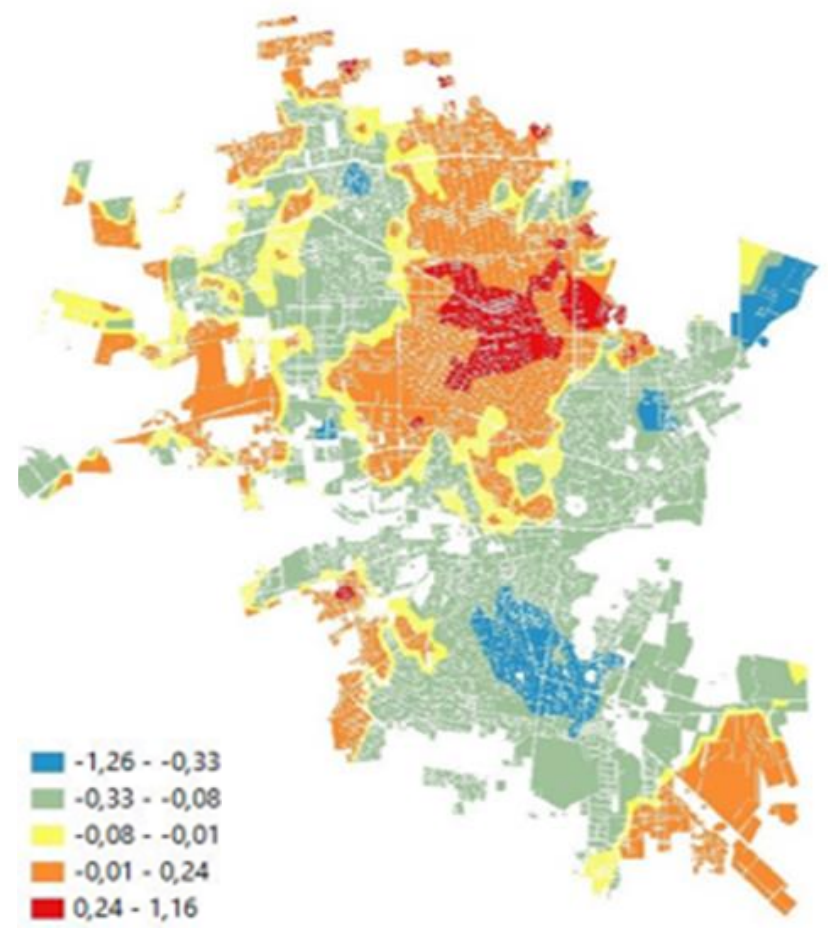

Fuente: Elaboración propia

Citación: QUINTANA, J. et al. El valor del suelo habitacional. En: Libro de proceedings, CTV 2018. XII Congreso Internacional Ciudad y Territorio Virtual. "Ciudades y Territorios Inteligentes". UNCuyo, Mendoza, 5-7 septiembre 2018. Barcelona: CPSV, 2018, p. 165-180. 
Se aplica el Índice de Moran a los residuos del modelo de precios hedónicos resuelto por OLS y no presentan una autocorrelación espacial significativa, sin embargo, la variable dependiente LN_V_U_SUELO si presentan el fenómeno de asociación espacial. En la ¡Error! No se encuentra el origen de la referencia., se muestra un p-valor inferior a 0.05 , con lo cual es muestra que la presencia de la auto correlación espacial positiva es significativa en más del $95 \%$.

\section{Figura 11. Auto correlación espacial valor del suelo}

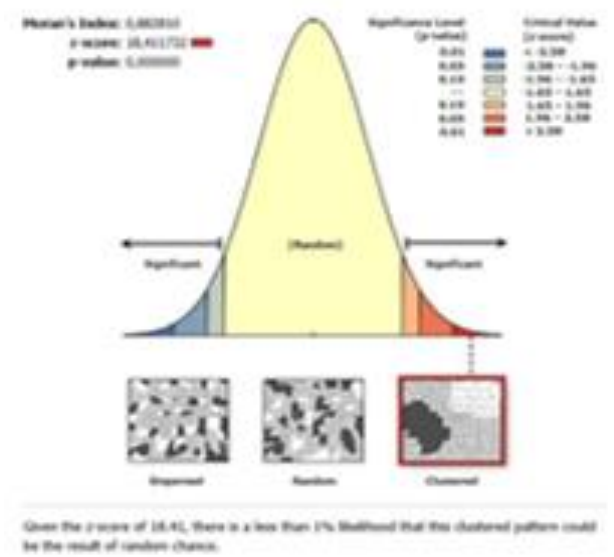

Fuente: Elaboración propia

Lo anterior sugiere una posible asociación espacial, por lo que se aplica la técnica de regresión geográficamente ponderada GWR, considerando una ponderación del tipo Gaussiano (kernel adaptativo) y un modelo de optimización por un número de vecinos de acuerdo con el Criterio de Información de Akaike (AICc).

La Tabla 4, muestra el número de vecinos promedio utilizado y sobre todo el valor de $\mathrm{R}^{2}$ ajustada con el $78.99 \%$, valor mayor que el obtenido a través de la regresión lineal múltiple, que proporcionó un $\mathrm{R}^{2}$ ajustada de $69.65 \%$. Así como el resto de los parámetros: cuadrado de los residuos, sigma, número efectivo y valor $\mathrm{AICc}$, todos ellos dentro de lo que se considera un buen modelo.

Tabla 4. Información de salida

\begin{tabular}{|c|c|c|}
\hline CONCEPTO & & INDICADOR \\
\hline Neighbors & & 61 \\
\hline ResidualSquares & & 15,499799 \\
\hline EffectiveNumber & & 29,277086 \\
\hline Sigma & & 0,2865831 \\
\hline AICc & & 95,688838 \\
\hline $\mathrm{R} 2$ & & 0,8172966 \\
\hline R2Adjusted & & 0,7899215 \\
\hline Dependent Field & LN_V_U_SUE & 0 \\
\hline Explanatory Field & FAC $\bar{C}$ REN & 1 \\
\hline Explanatory Field & FAC_AGUA & 2 \\
\hline
\end{tabular}

Fuente: Elaboración propia

En la Tabla 5, se presentan los valores principales obtenidos con la aplicación de la técnica GWR, en los primeros 21 puntos, de los 218 que componen la muestra.

Citación: QUINTANA, J. et al. El valor del suelo habitacional. En: Libro de proceedings, CTV 2018. XII Congreso Internacional Ciudad y Territorio Virtual. "Ciudades y Territorios Inteligentes". UNCuyo, Mendoza, 5-7 septiembre 2018. Barcelona: CPSV, 2018, p. 165-180. 
Libro de proceedings

Tabla 5. Modelo GWR a partir de los factores de renta y consumo de agua

\begin{tabular}{|c|c|c|c|c|c|c|c|c|}
\hline & $\underset{\text { bser }}{\text { Ln_V_U_O }}$ & Ind Cond & LocalR2 & $\begin{array}{c}\text { Ln_V_U } \\
\text { Predic }\end{array}$ & Intercept & $\underset{\text { EN }}{\text { C1_FAC_R }}$ & $\begin{array}{c}\text { C2_FAC_A } \\
\text { GU }\end{array}$ & Residual \\
\hline \multirow[t]{2}{*}{0} & 8,39000000 & 10,8051845 & 0,5187831 & 8,1290397 & 6,4099055 & 0,3196611 & 0,0363525 & 0,2609602 \\
\hline & 000 & 8500 & 5305 & 0244 & 5014 & 1304 & 9580 & 9756 \\
\hline \multirow[t]{2}{*}{1} & 8,50000000 & 10,8070264 & 0,4893246 & 8,1244174 & 6,4346068 & 0,2996835 & 0,0360296 & 0,3755825 \\
\hline & 000 & 7620 & 2876 & 8067 & 8209 & 7625 & 9227 & 1933 \\
\hline \multirow[t]{2}{*}{2} & 8,51000000 & 10,6220619 & 0,6183512 & 8,1038349 & 6,3493802 & 0,3955299 & 0,0356816 & 0,4061650 \\
\hline & 000 & 3600 & 8696 & 6916 & 1417 & 5349 & 4154 & 3084 \\
\hline \multirow[t]{2}{*}{3} & 6,91000000 & 24,7020803 & 0,2037566 & 6,5069274 & 7,7602560 & 0,1580276 & - & 0,4030725 \\
\hline & 000 & 1260 & 4815 & 8798 & 7201 & 2870 & $\begin{array}{r}0,0704688 \\
3502\end{array}$ & 1202 \\
\hline \multirow[t]{2}{*}{4} & 6,48000000 & 26,0344201 & 0,1950270 & 6,4525005 & 7,6318285 & 0,1149708 & - & 0,0274994 \\
\hline & 000 & 2210 & 9343 & 0965 & 0392 & 2486 & $\begin{array}{r}0,0675816 \\
3837\end{array}$ & 9035 \\
\hline \multirow[t]{2}{*}{5} & 6,56000000 & 24,3844450 & 0,2143354 & 6,7398341 & 7,6976095 & 0,1939333 & - & \\
\hline & 000 & 8360 & 6390 & 5086 & 6430 & 7008 & $\begin{array}{r}0,0640821 \\
7006\end{array}$ & $\begin{array}{r}0,1798341 \\
5086\end{array}$ \\
\hline \multirow[t]{2}{*}{6} & 6,51000000 & 23,6600688 & 0,2140433 & 6,7029055 & 7,6791961 & 0,1994630 & - & \\
\hline & 000 & 1840 & 7009 & 8621 & 8515 & 0032 & $\begin{array}{r}0,0623002 \\
2831\end{array}$ & $\begin{array}{r}0,1929055 \\
8621\end{array}$ \\
\hline \multirow[t]{2}{*}{7} & 6,69000000 & 21,9730545 & 0,2121643 & 6,5190335 & 7,6439923 & 0,2097206 & - & 0,1709664 \\
\hline & 000 & 4420 & 1010 & 3336 & 4677 & 1343 & $\begin{array}{r}0,0585697 \\
2317\end{array}$ & 6664 \\
\hline \multirow[t]{2}{*}{8} & 6,41000000 & 13,1547879 & 0,3348229 & 6,1226575 & 6,5781322 & 0,2425518 & 0,0038004 & 0,2873424 \\
\hline & 000 & 4550 & 4351 & 0188 & 4066 & 4292 & 8840 & 9812 \\
\hline \multirow[t]{2}{*}{9} & 6,46000000 & 12,9308786 & 0,3461386 & 6,3212616 & 6,5719007 & 0,2503453 & 0,0043981 & 0,1387383 \\
\hline & 000 & 1600 & 2145 & 1048 & 0084 & 0983 & 9704 & 8952 \\
\hline \multirow[t]{2}{*}{10} & 6,65000000 & 13,0328218 & 0,3371305 & 6,4058392 & 6,5742771 & 0,2442769 & 0,0040187 & 0,2441607 \\
\hline & 000 & 5770 & 5204 & 4698 & 3891 & 0462 & 3093 & 5302 \\
\hline \multirow[t]{2}{*}{11} & 6,66000000 & 13,3218655 & 0,3327 & 89571 & 6,5 & 0,2432 & 0,0030376 & 0,1610428 \\
\hline & 000 & 5190 & 0534 & 8 & 17 & 5738 & 6647 & 1581 \\
\hline \multirow[t]{2}{*}{12} & 6,52000000 & 25,7307856 & 0,2351 & 6,6010041 & 7,4454 & 0,1017789 & - & - \\
\hline & 000 & 6430 & 4372 & 8093 & 8623 & 3626 & $\begin{array}{r}0,0591881 \\
8342\end{array}$ & $\begin{array}{r}0,0810041 \\
8093\end{array}$ \\
\hline \multirow[t]{2}{*}{13} & 6,19000000 & 20,7357740 & 0,2473 & $6,4 \varepsilon$ & 6,6 & 0,2426 & 0,0003440 & . \\
\hline & 000 & 0120 & 0161 & 6308 & 4100 & 0171 & 0564 & $\begin{array}{r}0,2928759 \\
6308\end{array}$ \\
\hline \multirow[t]{2}{*}{14} & 6,59000000 & 23,0048516 & 0,2214729 & 6,6186250 & 7,6025225 & 0,2285026 & - & - \\
\hline & 000 & 5950 & 8603 & 6208 & 5535 & 0332 & $\begin{array}{r}0,0553632 \\
1749\end{array}$ & $\begin{array}{r}0,0286250 \\
6208\end{array}$ \\
\hline \multirow[t]{2}{*}{15} & 6,42000000 & 10,0674272 & $0,6 \mathrm{~s}$ & 6,7 & 5,9896 & 0,2562697 & 0,0467719 & \\
\hline & 000 & 3780 & 8862 & 93 & 792 & 4500 & 3429 & $\begin{array}{r}0,3258514 \\
9343\end{array}$ \\
\hline \multirow[t]{2}{*}{16} & 6,55000000 & 10,0172418 & 0,6922911 & 8376 & 6,0012992 & 0,2550496 & 0,0460447 & - \\
\hline & 000 & 6060 & 0420 & 9008 & 5716 & 5496 & 5335 & $\begin{array}{r}0,2508376 \\
9008\end{array}$ \\
\hline \multirow[t]{2}{*}{17} & 6,70000000 & 10,8085933 & 0,648 & 6 , & 59 & 0,2445 & 0,0465958 & 0,2619128 \\
\hline & 000 & 8650 & 9947 & 9162 & 6971 & 1368 & 3576 & 0839 \\
\hline \multirow[t]{2}{*}{18} & 6,14000000 & 11,9241461 & 0,3800575 & 6,3035221 & 6,4768690 & 0,2565896 & 0,0104683 & - \\
\hline & 000 & 1460 & 7488 & 9567 & 7217 & 2846 & 7611 & $\begin{array}{r}0,1635221 \\
9567\end{array}$ \\
\hline \multirow[t]{2}{*}{19} & 6,51000000 & 11,9248487 & 0,3806291 & 6,4779437 & 6,4781275 & 0,2572835 & 0,0104089 & 0,0320562 \\
\hline & 000 & 5730 & 1855 & 7458 & 3334 & 6640 & 7854 & 2542 \\
\hline \multirow[t]{2}{*}{20} & 6,26000000 & 12,1875517 & 0,3701932 & 6,2977258 & 6,5002137 & 0,2545525 & 0,0089603 & \\
\hline & 000 & 3200 & 7440 & 8907 & 7442 & 8069 & 4351 & $\begin{array}{r}0,0377258 \\
8907\end{array}$ \\
\hline
\end{tabular}

Fuente: Elaboración propia

Finalmente, en la Tabla 6, se comparan las características del modelo de regresión lineal múltiple, optimizado por mínimos cuadrados ordinarios (OLS) y el modelo GWR, donde se observan mejoras en los valores de $\mathrm{R}^{2}$, pero sobre todo, el valor de AICc que es menor en GWR, lo que implica la mejora del modelo GWR sobre el OLS. 
Tabla 6. Comparativa de resultados OLS y GWR

\begin{tabular}{llll}
\hline Modelo & $\mathbf{R}^{2}$ & $\mathbf{R}^{2}$ corregida & AICc \\
\hline OLS & 0.6993 & 0.6965 & 156.9688 \\
\hline GWR & 0.8173 & 0.7899 & 95.6888 \\
\hline
\end{tabular}

Fuente: Elaboración propia

\section{Conclusiones}

Los análisis de regresión lineal simple muestran como el valor del suelo puede ser explicado por el factor de educación en un $49 \%$ o por el de consumo de agua en un $49 \%$ o por el de renta en un $59 \%$, esto es, los factores de jerarquía social predominan en la formación del valor del suelo en la ciudad.

Los modelos obtenidos con la regresión lineal multivariada, muestran resultados que explica el valor del suelo en la ciudad, con un $69.65 \%$ de $\mathrm{R}^{2}$ ajustada, a partir de los factores de renta y consumo de agua de la población. Donde la principal variable asociada es la renta de la población; esto es, las personas se ubican principalmente en función de sus características económicas. En segundo lugar aparece el consumo de agua, como elemento asociado al valor del suelo.

El factor de educación por si solo tiene una explicación del $49 \%$ del valor del suelo, pero no aparece en el modelo final ya que presenta una fuerte correlación (0.901) con el factor de renta. Esto es el valor del suelo urbano habitacional en la ciudad de Hermosillo, está fuertemente asociado con la renta, la educación y el consumo de agua.

El análisis de regresión geográficamente ponderado, considera el efecto de asociación espacial de los valores en la ciudad, con lo cual es posible encontrar y concluir con modelos individuales que logran mejorar la explicación anterior. Esto es, se muestra un cierto grado de asociación espacial de los valores de suelo altos con altos y valores bajos con bajos.

\section{Bibliografía}

AGUAYO, E. y CHAPA, J. C. El robo a casa habitación en Monterrey, Nuevo León. ¿Un problema de localización? En: EconoQuantum, 9 (1): 189-201. 2012. [En línea] Disponible en: http://www.scielo.org.mx/scielo.php?script=sci arttext\&pid=S1870-66222012000100012

ÁREA DE GOBIERNO DE MEDIO AMBIENTE Y SERVICIOS DE LA CIUDAD DE MADRID. Plan Municipal de Gestión de la Demanda de Agua en la Ciudad de Madrid. Madrid: Área de Gobierno de Medio Ambiente y Servicios de la Ciudad de Madrid. 2005.

COLEGIO DE VALUADORES PROFESIONALES DEL ESTADO DE SONORA. Valores del suelo para la ciudad de Hermosillo, Sonora. Hermosillo: COVAPROES. 2013.

CONSEJO NACIONAL DE POBLACIÓN. Índice de marginación por entidad federativa y municipio 2010. [En línea] 13 de Marzo de 2010. DFsiponible en: http://conapo.gob.mx/en/CONAPO/Indices de Marginacion 2010 por entidad federativa y municipio 
COOK, P. J. The Demand and Supply of Criminal Opportunities. En: Crime and Justice, 7; 1-27. 1986. [En línea] Disponible en: https://www.jstor.org/stable/1147515

CORTÉS, Y. y PARRA, R. Narcomenudeo: un neologismo para describir la venta de estupefacientes. En: Revista Criminalidad, 53 (2): 37-71. 2011. [En línea] Disponible en: http://www.scielo.org.co/scielo.php?pid=S1794-31082011000200003\&script=sci abstract\&tlng=es

HINDELANG, M. J.; GOTTFREDSON, M. R. \& GAROFALO, J. Victims of personal crime - an empirical foundation for a theory of personal victimization. Cambridge MA: Ballinger Publishing Co.1978.

INSTITUTO MUNICIPAL DE PLANEACION URBANA. implanhermosillo.gob.mx. 18 de Octubre de 2006. [En línea] Fecha de Consulta: Noviembre de 2014 Disponible en: www.implanhermosillo.gob.mx

INSTITUTO NACIONAL DE ESTAdístiCA GEOGRAFíA. Censos y Conteos de Población y Vivienda. [En línea] en: http://www.inegi.org.mx/est/contenidos/proyectos/ccpv/cpv2010/Municipios.aspx. 2010

MORALES, E. E. Ponderación del costo en el sistema de transporte que determine una tarifa equitativa. Hermosillo: Universidad de Sonora. 2015.

OJEDA, A. Análisis Socioespacial del Consumo de Agua Doméstica en Hermosillo Sonora. Monterrey: Universidad Autónoma de Nuevo León. 2013.

SECRETARÍA DE SEGURIDAD PÚBLICA. 22 de Agosto de 2016. [En línea] Disponible en: https://drive.google.com/file/d/OB8wkB wp-pwZWTExbGtHMzNwVjA/view

SHANDAS, V. y PARANDVASH, G. Integrating Urban Form and Demographics in Waterdemand Management: An Empirical Case Study of Portland Oregon. En: Environment and Planning B: Planning and Design, 37, 1-18. 2009. [En línea] Disponible en: https://journals.sagepub.com/doi/abs/10.1068/b35036 DOI: https://doi.org/10.1068/b35036

URQUIJO, M. El 60\% de los robos se dan en cerradas. En: El imparcial, pág. 1. 2016. (15 de Octubre de 2016).

VILALTA, C. J. El robo de vehículos en la ciudad de México: patrones espaciales y series de tiempo. En: Gestión y Política Pública, XX (1): 97-139. 2011. [En línea] Disponible en: http://www.scielo.org.mx/scielo.php?script=sci arttext\&pid=S1405-10792011000100003

Citación: QUINTANA, J. et al. El valor del suelo habitacional. En: Libro de proceedings, CTV 2018. XII Congreso Internacional Ciudad y Territorio Virtual. "Ciudades y Territorios Inteligentes". UNCuyo, Mendoza, 5-7 septiembre 2018. Barcelona: CPSV, 2018, p. 165-180. 